\title{
Effectiveness of the MFAS method for retrieval of height profiles of speed and direction of the wind from measurements by a Windcube 200s lidar
}

\section{Anton Stephan, Norman Wildmann, Igor N. Smalikho}

Anton Stephan, Norman Wildmann, Igor N. Smalikho, "Effectiveness of the MFAS method for retrieval of height profiles of speed and direction of the wind from measurements by a Windcube 200s lidar," Proc. SPIE 10833, 24th International Symposium on Atmospheric and Ocean Optics: Atmospheric Physics, 1083353 (13 December 2018); doi: 10.1117/12.2504450 


\title{
Effectiveness of the MFAS method for retrieval of height profiles of speed and direction of the wind from measurements by a Windcube 200s lidar
}

\author{
Anton Stephan ${ }^{\mathrm{a}}$, Norman Wildmann ${ }^{\mathrm{a}}$, Igor N. Smalikho*b \\ ${ }^{a}$ Institute of Atmospheric Physics of the German Aerospace Center (DLR) \\ Münchener Straße 20, Oberpfaffenhofen, Wessling 82234 Germany \\ ${ }^{b}$ V.E. Zuev Institute of Atmospheric Optics SB RAS \\ 1, Akademik Zuev Square, Tomsk 634055, Russia
}

\begin{abstract}
The method of maximum of the function of accumulated spectra (MFAS) was used for the first time to estimate the wind velocity vector from measurements by a micropulsed coherent Doppler lidar (MPCDL) with conical scanning by the probing beam. In the experiment with a Windcube 200s MPCDL, it is found that the use of MFAS allows an increase in the maximum height of retrieval of the vertical profiles of the wind speed and direction by an average of $30 \%$ in comparison with the filtered sine-wave fitting.
\end{abstract}

Keywords: coherent Doppler lidar, velocity and direction of wind

\section{INTRODUCTION}

To obtain the information about the wind speed and direction from raw data measured by a coherent Doppler lidar, during measurement the conical scanning by the probing beam around the vertical axis is usually used. In this case, the elevation angle $\varphi$ is fixed, and the azimuth angle $\theta$ changes with a constant rate. The accuracy of lidar estimation of the wind velocity vector $\mathbf{V}=\left\{V_{z}, V_{x}, V_{y}\right\}$ depends mostly on the signal-to-noise ratio SNR and the method of data processing. The simplest method of estimation of the vector $\mathbf{V}$ components is the direct sine-wave fitting a (DSWF) [13], which is efficient only at zero probability $P_{b}$ of bad (false) estimate of the radial velocity. At low SNR, the probability $P_{b}$ becomes nonzero. For this case, the method of filtered sine-wave fitting (FSWF) and the method of maximum of the function of accumulated spectra (MFAS) were proposed in [3]. The FSWF and MFAS are widely used in practice for treatment of raw data measured by $2-\mu \mathrm{m}$ pulsed coherent lidars. Advantages of the FSWF method in comparison with DSWF were earlier demonstrated in experiments with micropulsed coherent Doppler lidars (MPCDL). According to the theory [3], MFAS provides an acceptable result at lower SNR than FSWF does. However, the MFAS method was not applied earlier to processing of data measured by MPCDL.

In this paper, we study the efficiency of the MFAS method for visualization of wind from measurements by the Windcube 200s MPCDL (made by Leosphere, France) [4] at the conical scanning by a probing beam.

\section{ESTIMATION OF WIND VECTOR FROM MEASUREMENTS BY A WINDCUBE 20OS LIDAR}

The Windcube 200s lidar falls in the family of all-fiber MPCDLs characterized by the relatively low energy of the probing pulse and the high pulse repetition frequency $f_{p}$. From raw data measured by conically scanning Windcube $200 \mathrm{~s}$ lidar, the power spectra of the lidar signal $\hat{S}(f ; R, \theta)$ are estimated, where $f$ is the frequency and $R$ is the distance between the lidar and the center of the probed volume.

*smalikho@iao.ru; phone +7 3822 493595; fax +7 3822492086

24th International Symposium on Atmospheric and Ocean Optics: Atmospheric Physics, edited by

Gennadii G. Matvienko, Oleg A. Romanovskii, Proc. of SPIE Vol. 10833, 1083353

(c) 2018 SPIE · CCC code: 0277-786X/18/\$18 - doi: 10.1117/12.2504450 
Let $Z\left(t_{i} ; n\right)$ be a series of signal readouts of analog-to-digital converter (ADC) during the MPCDL operation. Here, $t_{i}=i \delta t$ is the time counted from the instant of maximum of the power distribution of $n$-th probing pulse in the plane of the lidar telescope; $i=1,2,3, \ldots, I$ is the sample number; $\delta t=1 / B_{0}$ is the sampling interval; $B_{0}$ is the bandwidth of an real signal (doubled Nyquist frequency), and $n=0,1,2,3, \ldots$ is the shot number. In the signal processor, to obtain power spectra of lidar signal, the rectangular time window $W(t)$ with the width $T_{W}$ comparable with the pulse duration $\tau_{p}$, fast Fourier transformation, and accumulation of data are used. As a result of signal processor operation, we obtain estimates of the spectrum of lidar signal power for different distances $R_{k}$ and azimuth angles $\theta_{m}$. These estimates can be represented as

$$
S\left(f_{l} ; R_{k}, \theta_{m}\right)=\frac{1}{N_{a}} \sum_{n^{\prime}=1}^{N_{a}}\left|\sum_{i^{\prime}=0}^{I^{\prime}-1} W\left(i^{\prime} \delta t\right) Z\left(2 R_{k} / c-T_{W} / 2+i^{\prime} \delta t ; N_{a} m+n^{\prime}\right) \exp \left(-2 \pi j \frac{l i^{\prime}}{I^{\prime}}\right)\right|^{2},
$$

where $f_{l}=l \Delta f$ is frequency; $l=0,1,2, \ldots, L-1$ is the spectral channel number; $L=I^{\prime} / 2$ is the number of all spectral channels; $\Delta f=B_{0} / I^{\prime}$ is the width of a spectral channel; $R_{k}=R_{0}+k \Delta R ; k=0,1,2, \ldots, K-1 ; \Delta R$ is the range gate length; $\theta_{m}=m \Delta \theta ; m=0,1,2, \ldots, M-1 ; \Delta \theta=2 \pi / M=N_{a} \omega_{0} / f_{p}$ is the azimuth angle resolution; $N_{a}$ is the number of laser shots used for spectral accumulation (averaging); $W(t)=1$ at $t \leq T_{W}$ and $W(t)=0$ at $t>T_{W} ; c$ is the speed of light, and $j=\sqrt{-1}$. The obtained spectra include the useful and noise components. At long distances from the lidar in the absence of clouds at a path, the echo signal is very weak and the measured spectrum is a noise spectrum, which is denoted as $S_{N}\left(f_{l}, \theta_{m}\right)$. Then the estimate of the Doppler spectrum $S\left(f_{l} ; R_{k}, \theta_{m}\right)$ carrying the information about the velocity of scattering particles (transported by the air flow) can be represented in the form

$$
S_{D}\left(f_{l} ; R_{k}, \theta_{m}\right)=S\left(f_{l} ; R_{k}, \theta_{m}\right)-S_{N}\left(f_{l}, \theta_{m}\right) \text {. }
$$

Table 1. Parameters of Windcube 200s lidar.

\begin{tabular}{|l|l|}
\hline Wavelength $\lambda=1.543 \mu \mathrm{m}$ & $\begin{array}{l}\text { Number of spectral channels in the range } \\
0-125 \mathrm{MHz} L=32\end{array}$ \\
\hline Pulse energy $E_{p}=50 \mu \mathrm{J}$ & Velocity resolution $\Delta V=3 \mathrm{~m} / \mathrm{s}$ \\
\hline Pulse duration $\tau_{p}=200 \mathrm{~ns}$ & Number of pulses for accumulation $N_{a}=4000$ \\
\hline Pulse repetition frequency $f_{p}=20 \mathrm{kHz}$ & Minimum distance $R_{0}=100 \mathrm{~m}$ \\
\hline Intermediate frequency $f_{\text {int }}=69.3 \mathrm{MHz}$ & Range gate length $\Delta R=50 \mathrm{~m}$ \\
\hline Bandwidth $B_{0}=250 \mathrm{MHz}$ & Elevation angle $\varphi=35.3^{\circ}$ \\
\hline Width of the time window $T_{W}=144 \mathrm{~ns}$ & Azimuth angle resolution $\Delta \theta=1^{\circ}$ \\
\hline $\begin{array}{l}\text { Longitudinal dimension of the sensing volume } \\
\Delta z=36 \mathrm{~m}\end{array}$ & Duration of one conical scan $T_{\text {scan }}=72 \mathrm{~s}$ \\
\hline
\end{tabular}

The main parameters of the Windcube 200s lidar in the experiment, whose description can be found below, are given in Table 1. According to this table, the bandwidth $B_{0}=250 \mathrm{MHz}$, the number of spectral channels is $L=32$, and, consequently, the width of the spectral channel is $\Delta f=B_{0} /(2 L)=3.90625 \mathrm{MHz}$. This corresponds to the channel width in the units of velocity $\Delta V=(\lambda / 2) \Delta f \approx 3 \mathrm{~m} / \mathrm{s}(\lambda=1.543 \mu \mathrm{m}$ is the wavelength of probing radiation). At this quite rough frequency resolution, the radial velocity (projection of the wind velocity vector onto the optical axis of the probing beam) can be estimated from the spectrum $S_{D}\left(f_{l} ; R_{k}, \theta_{m}\right)$ with a large error. Therefore, using the Fourier interpolation, we pass from $S\left(f_{l} ; R_{k}, \theta_{m}\right), S_{N}\left(f_{l}, \theta_{m}\right)$, and $S_{D}\left(f_{l} ; R_{k}, \theta_{m}\right)$ to the corresponding spectra at the frequencies $f_{l^{\prime}}=l^{\prime} \delta f$, where $l^{\prime}=0,1,2, \ldots, L N_{F}-1, N_{F}=64$ and $\delta f=\Delta f / N_{F} \approx 0.061 \mathrm{MHz}$ (frequency of the spectral channel in the units of velocity $\delta V=(\lambda / 2) \delta f \approx 0.047 \mathrm{~m} / \mathrm{s})$. 
In the considered lidar, the master laser radiation is divided into the reference and probing beam. With an acousto-optical modulator, the probing beam frequency shifts by $f_{\text {int }}$, which is called an intermediate frequency (difference between frequencies of the reference and probing radiation). The position of the peak in the measured spectrum at the frequency $f_{\text {int }}$ corresponds to zero value of the radial velocity. With allowance for the Doppler equation, the radial velocity $\hat{V}_{r}\left(R_{k}, \theta_{m}\right)$ can be estimated from the spectrum $S_{D}\left(f_{l^{\prime}} ; R_{k}, \theta_{m}\right)$ in the frequency range $f_{l^{\prime}} \in\left[f_{\text {int }}-B / 2, f_{\text {int }}+B / 2\right]$, where $B=50 \mathrm{MHz}$, from the position of the spectral maximum, that is, $\max \left\{S_{D}\left(f_{l} ; R_{k}, \theta_{m}\right)\right\}=S_{D}\left(f_{\max } ; R_{k}, \theta_{m}\right)$ and

$$
\hat{V}_{r}\left(R_{k}, \theta_{m}\right)=(\lambda / 2)\left[f_{\max }\left(R_{k}, \theta_{m}\right)-f_{\text {int }}\right] .
$$

According to the data given in Table 1 for $T_{W}$ and $\tau_{p}$, the longitudinal dimension of the probed volume determined as $\Delta z=\left(c T_{W} / 2\right) / \operatorname{erf}\left(\sqrt{\ln 2} T_{W} / \tau_{p}\right)(\operatorname{erf}(x)$ is the standard error function) [5], is equal to $36 \mathrm{~m}$.

In addition to the radial velocity, the spectra $S_{D}\left(f_{l^{\prime}} ; R_{k}, \theta_{m}\right)$ and $S_{N}\left(f_{l^{\prime}}, \theta_{m}\right)$ can be used to estimate the signal-to-noise ratio $\operatorname{SNR}\left(R_{k}, \theta_{m}\right)$ defined as the ratio of the average heterodyne signal power to the average detector noise power in a bandwidth $B=50 \mathrm{MHz}$ as follows:

$$
\operatorname{SNR}\left(R_{k}, \theta_{m}\right)=\sum_{l^{\prime}=l_{-}}^{l_{+}} S_{D}\left(f_{l^{\prime}} ; R_{k}, \theta_{m}\right) / \sum_{l^{\prime}=l_{-}}^{l_{+}} S_{N}\left(f_{l^{\prime}} ; \theta_{m}\right),
$$

where $l_{ \pm}=\left[\left(f_{\text {int }} \pm B / 2\right) / \delta f\right]$ and square brackets denote rounding up or down to the nearest integer number.
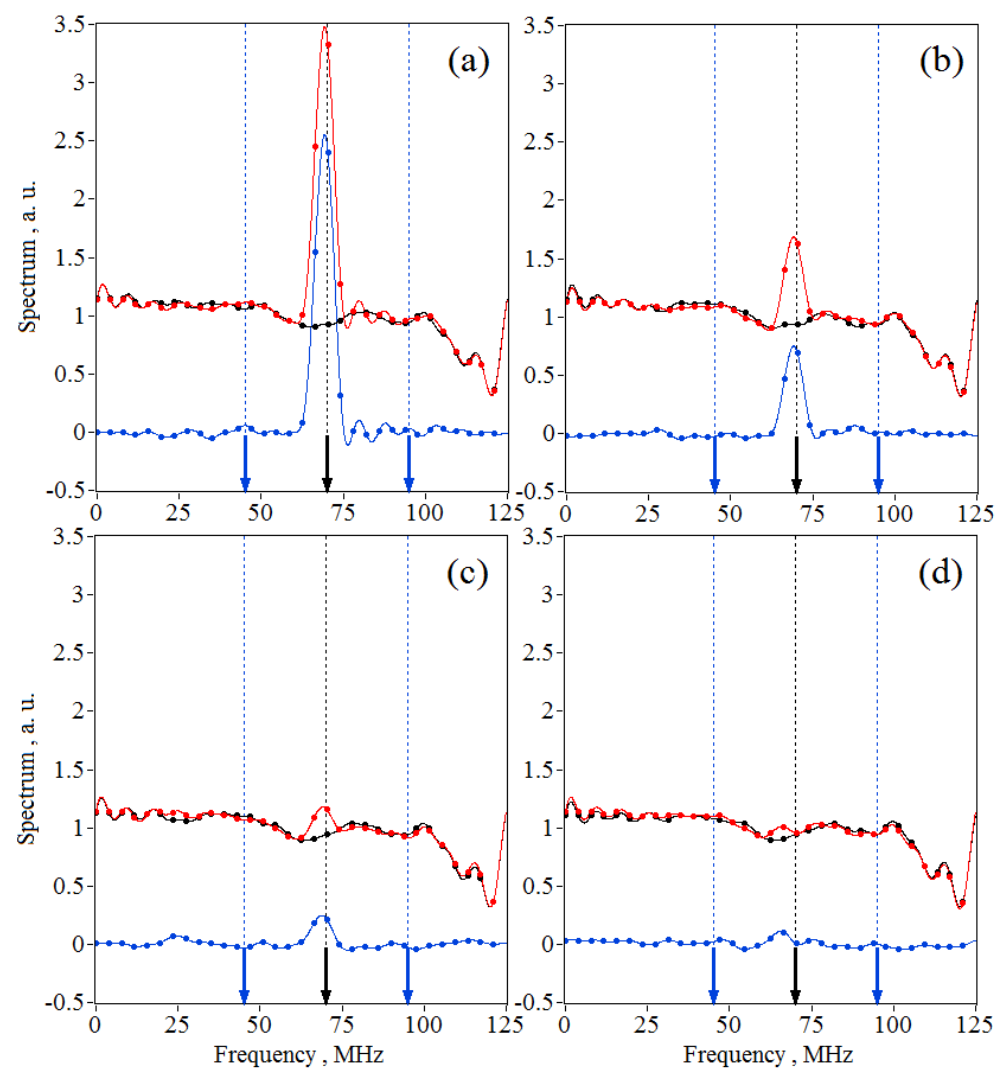

Figure 1. Initial spectra $S\left(f_{l} ; R_{k}, \theta_{m}\right), S_{N}\left(f_{l} ; \theta_{m}\right)$, and $S_{D}\left(f_{i} ; R_{k}, \theta_{m}\right)$ (respectively, red, black, and blue circles) and spectra after the Fourier interpolation $S\left(f_{l} ; R_{k}, \theta_{m}\right), S_{N}\left(f_{l} ; \theta_{m}\right)$, and $S_{D}\left(f_{l} ; R_{k}, \theta_{m}\right)$ (respectively, red, black, and blue curves) obtained from measurements by the Windcube 200s lidar at $\mathrm{SNR}=-5 \mathrm{~dB}(\mathrm{a}),-10 \mathrm{~dB}(\mathrm{~b}),-15 \mathrm{~dB}(\mathrm{c})$, and $-20 \mathrm{~dB}(\mathrm{~d})$. Blue arrows indicate the limits of the 50-Mhz passband, and the black arrow shows the position of the intermediate frequency. 
Figure 1 shows examples of initial spectra (without Fourier interpolation) $S\left(f_{l} ; R_{k}, \theta_{m}\right), S_{N}\left(f_{l} ; \theta_{m}\right)$, and $S_{D}\left(f_{l} ; R_{k}, \theta_{m}\right)$ and the same spectra after Fourier interpolation $S\left(f_{l^{\prime}} ; R_{k}, \theta_{m}\right), S_{N}\left(f_{l^{\prime}} ; \theta_{m}\right), S_{D}\left(f_{l^{\prime}} ; R_{k}, \theta_{m}\right)$ obtained from measurements by the Windcube 200s lidar at different signal-to-noise ratio determined by Eq. (4). It should be noted that here the values of the spectra in the zeroth and first channels $(l=0$ and $l=1)$ were replaced with the values from the second channel $(l=2)$. It can be seen that as SNR decreases, the height of the spectral peak carrying the information about the radial wind velocity decreases too. Even at $\mathrm{SNR}=-20 \mathrm{~dB}$, the estimate of the radial velocity $\hat{V}_{r}\left(R_{k}, \theta_{m}\right)$ can be obtained with the rather high accuracy owing to the large number of pulses used for spectral accumulation $\left(N_{a}=4000\right.$, see Table 1).

If the array of estimates of the radial velocity $\hat{V}_{r}\left(R_{k}, \theta_{m}\right)$ obtained from lidar measurements for one complete conical scan $\left(0^{\circ} \leq \theta_{m}<360^{\circ}\right)$ contains no bad estimates or the probability of $P_{b}$ does not exceed $1 \%$, then for determination of the wind velocity vector $\mathbf{V}=\left\{V_{z}, V_{x}, V_{y}\right\}$ ( $V_{z}$ is the vertical component of the wind vector, $V_{x}$ and $V_{y}$ are horizontal components) one can use the direct sine-wave fitting (DSWF) [1-3]. On the assumption of statistical homogeneity of the horizontal wind, the DSWF method consists in minimization of the functional

$$
\rho(\mathbf{V})=\sum_{m=0}^{M-1}\left[V_{r}\left(R_{k}, \theta_{m}\right)-\mathbf{S}\left(\theta_{m}\right) \cdot \mathbf{V}\right]^{2},
$$

where $\mathbf{S}\left(\theta_{m}\right)=\left\{\sin \varphi, \cos \varphi \cos \theta_{m}, \cos \varphi \sin \theta_{m}\right\}$ is the unit vector along the direction of the $m$-th ray. If the estimate of the wind velocity vector is denoted as $\hat{\mathbf{V}}=\left\{\hat{V}_{z}, \hat{V}_{x}, \hat{V}_{y}\right\}$, we have $\min \{\rho(\mathbf{V})\}=\rho(\hat{\mathbf{V}})$. The procedure of minimization of functional (5) reduces to solution of the system of linear equations: $\nabla \rho(\mathbf{V})=0$. Here, the operator is $\nabla=\left\{\partial / \partial V_{z}, \partial / \partial V_{x}, \partial / \partial V_{y}\right\}$.

At the extremely low signal-to-noise ratio, the position of the spectral maximum $S_{D}\left(f_{l^{\prime}} ; R_{k}, \theta_{m}\right)$ may be associated, with some probability, with fluctuations of the noise component of the spectrum $S_{N}\left(f_{l^{\prime}} ; \theta_{m}\right)-<S_{N}\left(f_{l^{\prime}} ; \theta_{m}\right)>$ (angular brackets denote the ensemble averaging). Taking into account that in the frequency range $f_{l^{\prime}} \in\left[f_{\text {int }}-B / 2, f_{\text {int }}+B / 2\right]$ the spectrum $S_{N}\left(f_{l^{\prime}} ; \theta_{m}\right)$ is close to the white noise (see black curves in Fig. 1), bad estimates of the radial velocity (or false estimate associated with a noise peak) can range from $-\lambda B / 4$ to $+\lambda B / 4$ (from $-19.3 \mathrm{~m} / \mathrm{s}$ to $+19.3 \mathrm{~m} / \mathrm{s}$ ) with approximately identical probability. In this case, the array $\hat{V}_{r}\left(R_{k}, \theta_{m}\right)$ contains a large percentage of bad estimates. Therefore, to determine the wind velocity vector from this array, the filtering of good estimates of the radial velocity is required. In [3], it was proposed to determine the wind velocity vector by the method of filtered sine-wave fitting (FSWF). This method consists essentially in the following. From the obtained array of estimates of radial velocities $\hat{V}_{r}\left(R_{k}, \theta_{m}\right)$, the function of three variables $V_{z}, V_{x}$, and $V_{y}$ is calculated consequently for every height $h_{k}=R_{k} \sin \varphi$ :

$$
Q\left(\mathbf{V} ; h_{k}\right)=\frac{1}{M} \sum_{m=0}^{M-1} \exp \left\{-\left[\hat{V}_{r}\left(R_{k}, \theta_{m}\right)-\mathbf{S}\left(\theta_{m}\right) \cdot \mathbf{V}\right]^{2} /\left(2 \sigma_{g}^{2}\right)\right\},
$$

where $\sigma_{g}$ is the filtering parameter specified according to the instrumental broadening of the Doppler spectrum and inhomogeneity of the wind field. The estimate of the wind vector $\hat{\mathbf{V}}$ is determined from the position of maximum of this function:

$$
\max \left\{Q\left(\mathbf{V} ; h_{k}\right)\right\}=Q\left(\hat{\mathbf{V}} ; h_{k}\right) .
$$

In comparison with DSWF, the FSWF method allows us to obtain an acceptable result at the much lower signal-to-noise ratio SNR, which was demonstrated, in particular, in [3].

Another method to determine the wind velocity vector from strongly noisy lidar data is the method of maximum of the function of accumulated spectra (MFAS). From the array of Doppler spectra obtained after the Fourier interpolation 
$S_{D}\left(f_{l^{\prime}} ; R_{k}, \theta_{m}\right)$ at $f_{l^{\prime}} \in\left[f_{\text {int }}-B / 2, f_{\text {int }}+B / 2\right]$, the function of accumulated spectra $F_{a}\left(\mathbf{V} ; h_{k}\right)$ is calculated for every height $h_{k}$ as:

$$
F_{a}\left(\mathbf{V} ; h_{k}\right)=\frac{1}{M} \sum_{m=0}^{M-1} S_{D}\left(l^{\prime}\left(\theta_{m}, \mathbf{V}\right) \delta f ; R_{k}, \theta_{m}\right),
$$

where $l^{\prime}\left(\theta_{m}, \mathbf{V}\right)$ is $\left(f_{\text {int }}+(2 / \lambda) \mathbf{S}\left(\theta_{m}\right) \cdot \mathbf{V}\right) / \delta f$ rounded up or down to the nearest integer number. The estimate of the vector $\hat{\mathbf{V}}$ is determined from the position of maximum of this function:

$$
\max \left\{F_{a}\left(\mathbf{V} ; h_{k}\right)\right\}=F_{a}\left(\hat{\mathbf{V}} ; h_{k}\right) .
$$

In contrast to the DSWF and FSWF methods, the MFAS method does not require obtaining estimates of the radial wind velocity. Summation in Eq. (8) means the additional accumulation of spectra, which leads to averaging of fluctuations of the noise component of the spectrum and, consequently, to an increase in the probability that the signal peak exceeds all noise peaks in the accumulated spectrum. At $N_{a}=4000$ and $M=360$, the total number of pulses used for accumulation of data of one complete conical scan is $N_{a} M=1440000$. For some SNR values, the point of maximum of the function $F_{a}\left(\mathbf{V} ; h_{k}\right)$ is the sought wind velocity vector. We have examined the efficiency of the MFAS method in the numerical and field experiments.

\section{NUMERICAL EXPERIMENT}

We have conducted numerical simulation of the measurement by the Windcube 200s lidar when the conical scanning by the probing beam is used. The parameters of this lidar can be found in Table 1. To obtain Doppler spectra carrying the information about the wind at the distance $R$ from the lidar, samples of lidar signal $Z\left(t_{i} ; n\right)$ and noise $Z_{N}\left(t_{i} ; n\right)$ (signals recorded by the lidar from long distances) were generated at the computer by the following algorithm [5-7]:

$$
Z\left(t_{i} ; n\right)=\operatorname{Re}\left\{Z_{S}\left(t_{i} ; n\right)\right\}+Z_{N}^{\prime}\left(t_{i} ; n\right),
$$

where

$$
Z_{S}\left(t_{i} ; n\right)=\sqrt{\operatorname{SNR} \frac{2 B}{B_{0}} \frac{\delta R}{\sqrt{\pi} \Delta p}} \sum_{i^{\prime}=0}^{N_{p}-1} \zeta\left(i+i^{\prime} ; n\right) A\left(i^{\prime}\right) \exp \left\{2 \pi j t_{i}\left[f_{\text {int }}+(2 / \lambda) V_{r}\left(R_{i+i^{\prime}}, \theta_{n}\right)\right]\right\}
$$

is a complex echo signal; $\quad \delta R=c \delta t / 2 ; \quad \Delta p=c \sigma_{p} / 2 ; \quad \sigma_{p}=\tau_{p} /(2 \sqrt{\ln 2}) ; \quad \zeta(i ; n)=\xi_{1}(i ; n)+j \xi_{2}(i ; n)$;

$A(i)=\exp \left\{-\left[\left(i-N_{p} / 2\right) \delta R / \Delta p\right]^{2} / 2\right\} ; V_{r}\left(R_{i}, \theta_{n}\right)=<V_{r}\left(R_{i}, \theta_{n}\right)>+V_{r}^{\prime}\left(R_{i}, \theta_{n}\right)$ is the radial velocity at the point with the coordinates $R_{i} \mathbf{S}\left(\theta_{n}\right) ; \quad V_{r}^{\prime}=V_{r}-<V_{r}>$ are turbulent fluctuations of the radial velocity; $R_{i}=R+\left(i-I_{W} / 2\right) \delta R$ is the distance from the lidar; $\theta_{n}=n \omega_{0} / f_{p}$ is the azimuth angle; $Z_{N}^{\prime}\left(t_{i} ; n\right)=\xi_{3}(i ; n)$ is the noise component of the signal coming from the probed volume centered at the distance $R$, and $Z_{N}\left(t_{i} ; n\right)=\xi_{4}(i ; n)$ is the lidar signal carrying only noise.

In this simulation algorithm, independent series of pseudorandom parameters $\xi_{1}(i ; n), \xi_{2}(i ; n), \xi_{3}(i ; n)$, and $\xi_{4}(i ; n)$ having properties of white noise with the Gaussian probability density function, zero mean, and unit variance are generated by the computer. If the wind field is assumed statistically homogeneous, the radial velocity $V_{r}\left(R_{i}, \theta_{n}\right)$ can be represented as

$$
V_{r}\left(R_{i}, \theta_{n}\right)=<V_{z}>\sin \varphi+<V_{x}>\cos \varphi \cos \theta_{n}+<V_{y}>\cos \varphi \sin \theta_{n}+V_{r}^{\prime}\left(R_{i}, \theta_{n}\right),
$$

where the average values of components of the wind velocity vector are constant. We have conducted the numerical experiment using the data of Table 1 for the case of very weak wind turbulence, when we can take $V_{r}^{\prime}\left(R_{i}, \theta_{n}\right)=0$ in Eq. (12), at $\left\langle V_{z}\right\rangle=0,\left\langle V_{x}\right\rangle=10 \mathrm{~m} / \mathrm{s},\left\langle V_{y}\right\rangle=0$, and the signal-to-noise ratio $\mathrm{SNR} \leq-27 \mathrm{~dB}$. Samples of the signal 
$Z\left(t_{i} ; n\right)$ and noise $Z_{N}\left(t_{i} ; n\right)$ simulated at fixed SNR (distance $R_{k}$ ) were used to calculate the spectra $S\left(f_{l} ; \theta_{m}\right)$, $S_{N}\left(f_{l}, \theta_{m}\right)$, and $S_{D}\left(f_{l} ; \theta_{m}\right)$ by Eqs. (1) and (2) with the following application of Fourier interpolation to $S_{D}\left(f_{l} ; \theta_{m}\right)$.

At the so low signal-to-noise ratio ( SNR $\leq-27 \mathrm{~dB}$ ), DSWF yields the definitely inacceptable result (strongly biased estimate of the wind velocity) [3, 5]. Therefore, for processing of simulated data, we use the FSWF and MFAS methods described by Eqs. (6)-(7) and (8)-(9), respectively. From the data simulated for different values of the signal-to-noise ratio SNR with the use of 1000 independent realizations (conical scans), we have calculated the probability $P_{<2}$ that the conditions $\left|\hat{V}_{x}-V_{x}\right|<2 \mathrm{~m} / \mathrm{s}$ and $\left|\hat{V}_{y}-V_{y}\right|<2 \mathrm{~m} / \mathrm{s}$ are fulfilled simultaneously, as well as the standard (root-meansquare) error of estimation of the wind velocity vector, which is defined as $E_{V}=\sqrt{\left\langle\left(\hat{V}_{x}-V_{x}\right)^{2}+\left(\hat{V}_{y}-V_{y}\right)^{2}\right\rangle}$, where $\left\{\hat{V}_{x}, \hat{V}_{y}\right\}$ is the estimate, and $\left\{V_{x}, V_{y}\right\}$ is the preset (true) value of the wind velocity vector.

Table 2. Probability $P_{<2}$ and error $E_{V}$ of lidar estimate of the wind velocity vector.

\begin{tabular}{|c|c|c|c|c|c|c|c|}
\hline Method & $\mathrm{SNR}, \mathrm{dB}$ & -32 & -31 & -30 & -29 & -28 & -27 \\
\hline \multirow{3}{*}{ FSWF } & $P_{<2}, \%$ & 32 & 49 & 71 & 88 & 97 & 100 \\
\cline { 2 - 7 } & $E_{V}, \mathrm{~m} / \mathrm{s}$ & 11.2 & 9.7 & 6.1 & 4.0 & 1.5 & 0.7 \\
\hline \multirow{2}{*}{ MFAS } & $P_{<2}, \%$ & 72 & 89 & 99 & 100 & 100 & 100 \\
\cline { 2 - 7 } & $E_{V}, \mathrm{~m} / \mathrm{s}$ & 6.6 & 3.8 & 1.4 & 0.7 & 0.55 & 0.4 \\
\hline
\end{tabular}

Table 2 presents the results of calculation of the probability $P_{<2}$ and the error $E_{V}$ for different values of the signal-tonoise ratio. One can see that if the FSWF method is used for processing of lidar data, the probability of acceptable estimate of the wind vector no lower than $99 \%$ can be obtained when the SNR exceeds approximately $-27.5 \mathrm{~dB}$. At the same time, the MFAS method allows the result with this accuracy to be obtained at SNR no lower than $-30 \mathrm{~dB}$. Thus, as follows from the results of numerical simulation, the MFAS method allows correct estimates of the wind velocity vector to be obtained at SNR lower by $2.5 \mathrm{~dB}$ (1.8 times smaller) than in the case of using the FSWF method.

\section{FIELD EXPERIMENT}

In October 2017, we have conducted the lidar experiment in Oberpfaffenhofen. The Windcube 200s lidar was installed on the roof of the building of the Institute of Atmospheric Physics of the German Aerospace Center (see Fig.2). Continuous measurements by this lidar with the use of conical scanning at the elevation angle $\varphi=35.3^{\circ}$ were conducted from 12:00 (Local Time hereinafter) on October 16 to 08:00 on October 19 of 2017. The parameters of the lidar, measurement, and treatment of data were the same as in the numerical experiment (see Table 1).

From the obtained spectra $S_{D}\left(f_{l^{\prime}} ; R_{k}, \theta_{m}\right)$ and $S_{N}\left(f_{l^{\prime}}, \theta_{m}\right)$, we determined the signal-to-noise ratio $\operatorname{SNR}\left(R_{k}, \theta_{m}\right)$ by Eq. (4). If $\mathrm{SNR}<<1$, the relative error of estimation of the signal-to-noise ratio $E_{\mathrm{SNR}}=\sqrt{\left\langle(\mathrm{SNR} / \mathrm{SNR}-1)^{2}\right\rangle}$ is described by the following equation [5]

$$
E_{\mathrm{SNR}}=\left(\sqrt{T_{W} B N_{a}} \mathrm{SNR}\right)^{-1}
$$

where SNR is the true signal-to-noise ratio. With allowance that $T_{W}=144 \mathrm{~ns}, B=50 \mathrm{MHz}$, and $N_{a}=4000$ (see Table 1), $E_{\mathrm{SNR}}$ does not exceed 0.3 provided that SNR $\geq 0.0196$ (-17 dB). According to the data of Table 2, the MFAS method allows an acceptable result to be obtained at $\mathrm{SNR} \geq-30 \mathrm{~dB}$. To determine the signal-to-noise ratio equal to $-30 \mathrm{~dB}$ with the relative error $E_{\mathrm{SNR}}$ no higher than 0.3 , it is necessary to average all estimates $\operatorname{SNR}\left(R_{k}, \theta_{m}\right)$ from measurements for one complete conical scan, that is, to obtain the average estimate as

$$
\operatorname{SN} \mathrm{N}\left(R_{k}\right)=M^{-1} \sum_{m=0}^{M-1} \operatorname{SNR}\left(R_{k}, \theta_{m}\right)
$$


With this averaging, the relative error $E_{\mathrm{SNR}}$ decreases $\sqrt{M}$ times, where the number of rays is $M=360$ (resolution in the azimuth angle $\Delta \theta=1^{\circ}$, see Table 1 ).

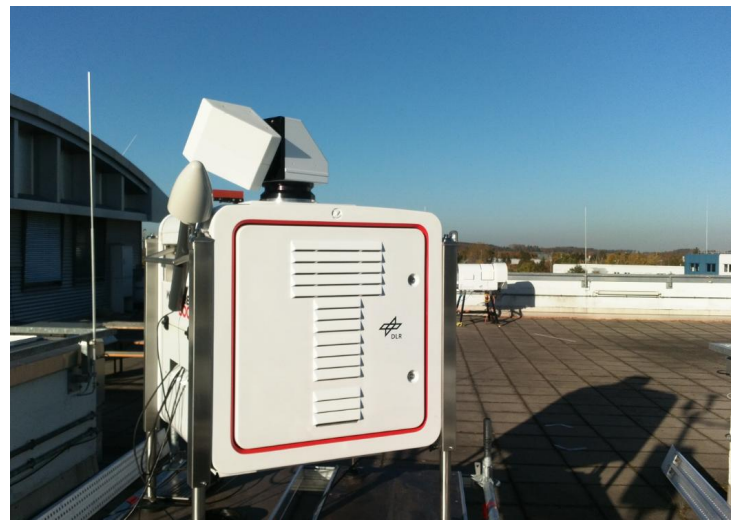

Figure 2. During the lidar experiment carried out in Oberpfaffenhoven from 10/16/2017 to 10/19/2017. The Windcube 200s lidar was installed on the roof of the building of the Institute of Atmospheric Physics of the German Aerospace Center (DLR).

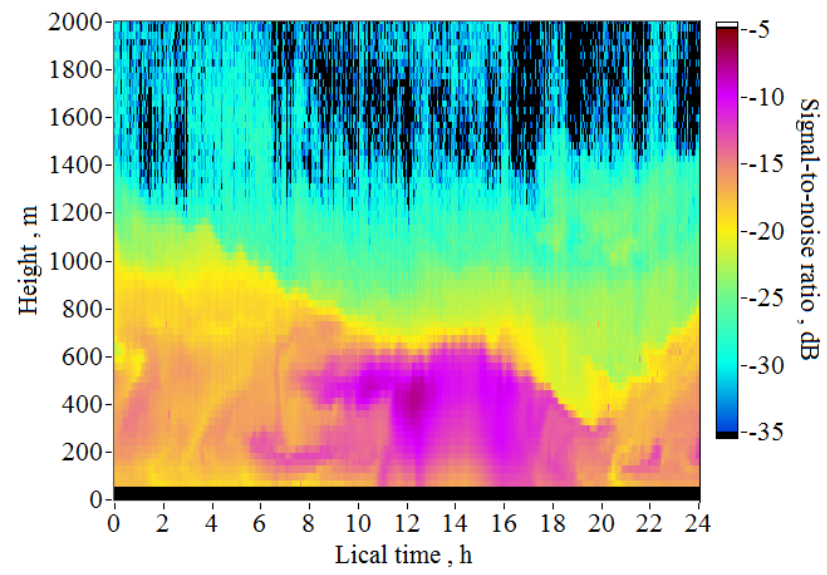

Figure 3. Height and time distribution of the signal-to-noise ratio obtained from measurements by the Windcube 200s lidar in Oberpfaffenhofen on October 17 of 2017.

To obtain estimates of the wind velocity vector $\hat{\mathbf{V}}=\left\{\hat{V}_{z}, \hat{V}_{x}, \hat{V}_{y}\right\}$ from measurements by the Windcube 200s lidar, we used the DSWF, FSWF, and MFAS methods. The wind speed $U$ and wind direction angle $\theta_{V}$ were calculated as $U=\left|\hat{V}_{x}+j \hat{V}_{y}\right|$ and $\theta_{V}=\arg \left\{\hat{V}_{x}+j \hat{V}_{y}\right\}$. Consequently, from the data of every conical scan, we obtained the estimates $\mathrm{S} \overline{\mathrm{N}} \mathrm{R}, U, \theta_{V}$, and $\hat{V}_{z}$ at different heights $h_{k}=R_{k} \sin \varphi=h_{0}+k \Delta h$. According to the data of Table 1, the initial height (above the lidar level) was $h_{0}=57.8 \mathrm{~m}$ and the height step was $\Delta h=\Delta R \sin \varphi=28.9 \mathrm{~m}$. Once the latest conical scan was accomplished, the next scan started $0.1 \mathrm{~s}$ later. The duration of one conical scan determined as $T_{\text {scan }}=M \Delta t$, where $M=360$ and $\Delta t=N_{a} / f_{p}=0.2 \mathrm{~s}$, was $72 \mathrm{~s}$. With the algorithms described above, we used the Windcube 200s lidar measurements to perform the spatiotemporal visualization of the signal-to-noise ratio and wind, that is, to obtain twodimensional distributions $\operatorname{SNR}\left(h_{k}, t_{n}\right), U\left(h_{k}, t_{n}\right), \theta_{V}\left(h_{k}, t_{n}\right)$, and $\hat{V}_{z}\left(h_{k}, t_{n}\right)$. Here, the time is $t_{n}=t_{0}+n T_{\text {scan }}$ and $n=0,1,2, \ldots$. 
Figure 3 shows the result of the spatiotemporal visualization for the signal-to-noise ratio $\mathrm{S} \overline{\mathrm{N}} \mathrm{R}\left(h_{k}, t_{n}\right)$ from the measurements by the Windcube 200s lidar on October 17 of 2017. One can see that the level of the lidar echo signal varied significantly for 24 hours. The relative error of estimates of the signal-to-noise ratio does not exceed $10 \%$ at heights no lower than $900 \mathrm{~m}$. According to the numerical simulation, the DSWF method yields an acceptable result at SNR $\geq-20 \mathrm{~dB}$, whereas the FSWF and MFAS provide acceptable results at SNR $\geq-27.5 \mathrm{~dB}$ and $\mathrm{SNR} \geq-30 \mathrm{~dB}$, respectively (see Table 2). Then, based on the data of Fig. 3, we can expect a significant increase of the maximal height of wind profile reconstruction with the use of FSWF and MFAS in comparison with DSWF.
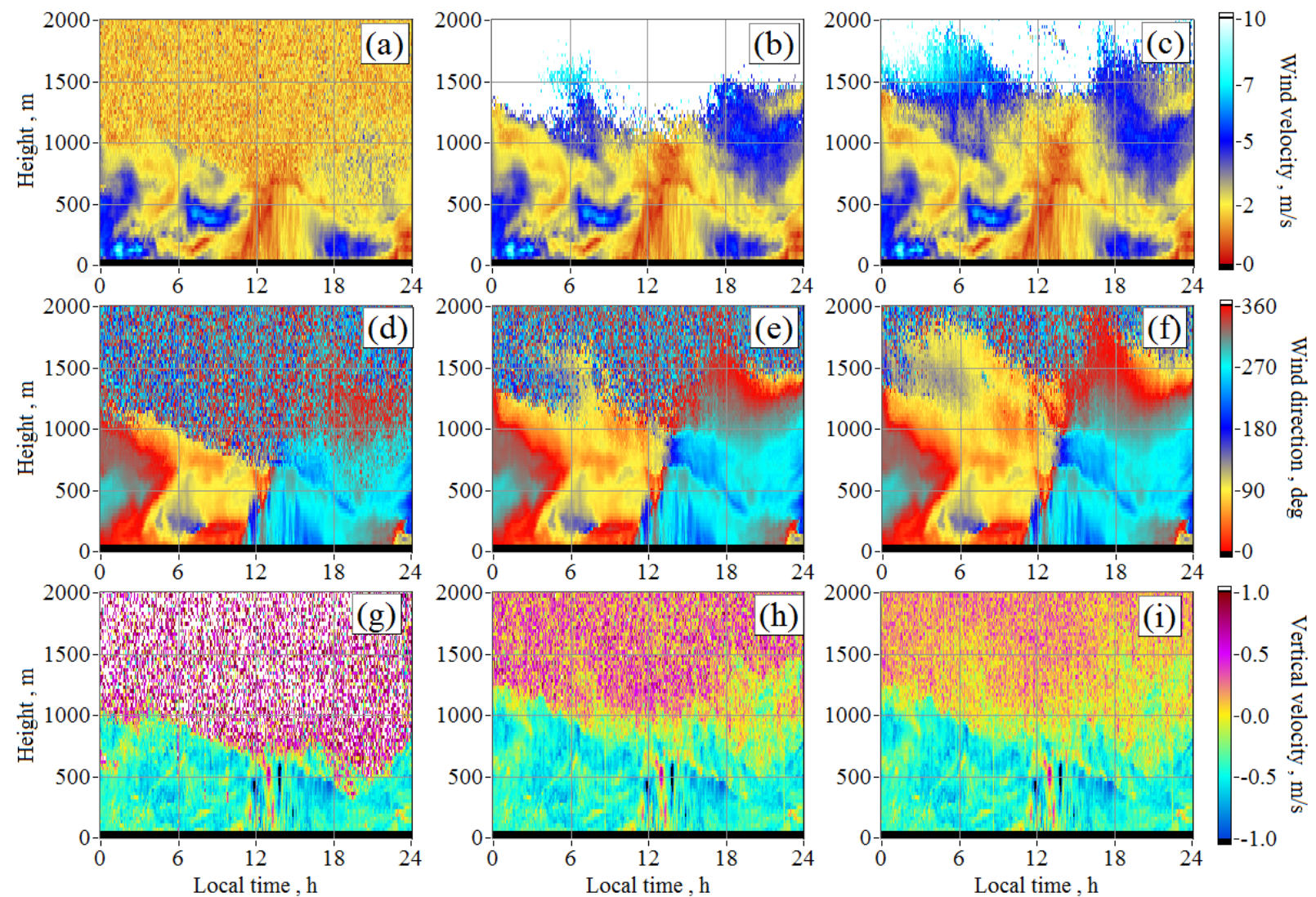

Figure 4. Height and time distributions of the wind speed (a, b, c), wind direction angle (d, e, f), and vertical component of the wind vector $(\mathrm{g}, \mathrm{h}, \mathrm{i})$ obtained from measurements by the Windcube 200s lidar in Oberpfaffenhofen on October 17 of 2017 with the use of the DSWF (a, d, g), FSWF (b, e, h), and MFAS (c, f, i) methods.

Figure 4 depicts the distributions of the wind velocity $U\left(h_{k}, t_{n}\right)$, wind direction angle $\theta_{V}\left(h_{k}, t_{n}\right)$, and vertical component of the wind vector $\hat{V}_{z}\left(h_{k}, t_{n}\right)$ obtained from the Windcube 200s measurements on October 17 of 2017 by three methods: DSWF, FSWF, and MFAS. From this figure, we can only visually judge the possibilities of the methods from the areas, within which the obtained distributions are smooth. It is obvious that sharp changes in estimates of the wind direction (to the opposite) with height or time are indicative of the large error. It can be seen that the largest area of the smooth distribution is inherent of MFAS, while DSWF is characterized by its smallest value.

Since we do not know the true value of wind, we take the wind vector $\overline{\mathbf{V}}=\left\{\bar{V}_{z}, \bar{V}_{x}, \bar{V}_{y}\right\}$ obtained by the MFAS method and averaged over $N^{\prime}=25$ scans (30-min averaging) with the data filtering as the true wind. That is, by analogy with Eqs. (6) - (7), we use the following algorithm: 


$$
\max \left\{Q_{V}\left(\mathbf{V} ; h_{k}, t_{n}\right)\right\}=Q\left(\overline{\mathbf{V}} ; h_{k}, t_{n}\right),
$$

where

$$
Q_{V}\left(\mathbf{V} ; h_{k}, t_{n}\right)=\sum_{n^{\prime}=0}^{N^{\prime}} \exp \left\{-\left[\hat{\mathbf{V}}^{(3)}\left(h_{k}, t_{n}+\left(n^{\prime}-N^{\prime} / 2\right) T_{\text {scan }}\right)-\mathbf{V}\right]^{2} /\left(2 \sigma_{g}^{2}\right)\right\}
$$

and $\hat{\mathbf{V}}^{(3)}\left(h_{k}, t_{n}\right)$ is the estimate of the wind velocity obtained from the lidar measurements at one $(n-$ th) scan with the use of the MFAS method.
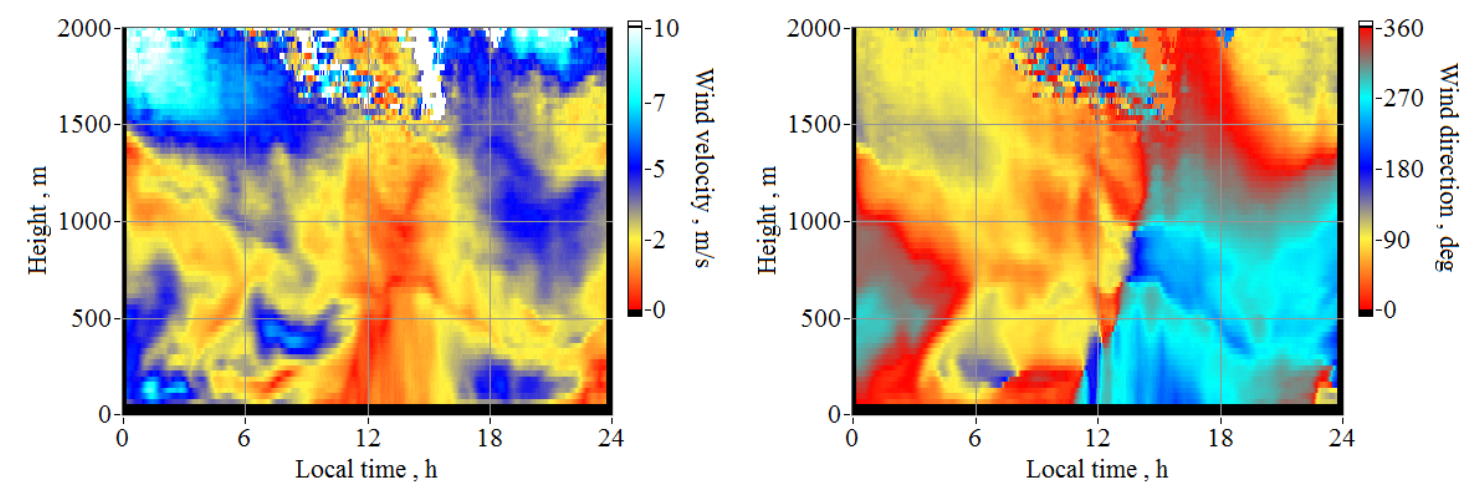

Figure 5. Distributions of wind velocity and wind direction angle obtained from data of Fig. 4(c,f) upon application of the procedure described by Eqs. (15) and (16).

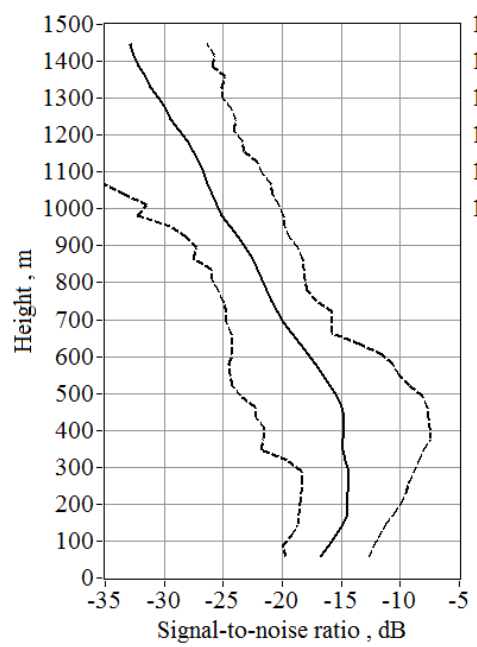

(a)

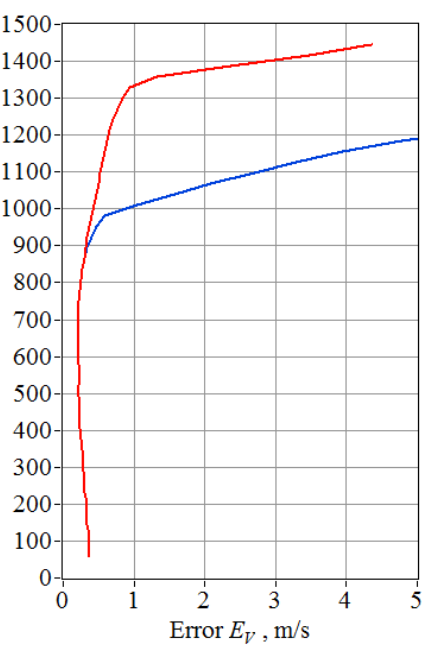

(b)

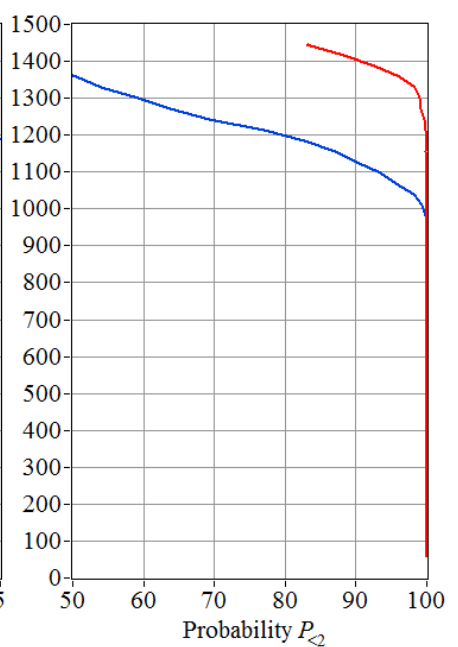

(c)

Figure 6. Vertical profiles of SNR (a), $E_{V}$ (b), and $P_{<2}$ (c) obtained from 24-h measurements by the Windcube 200s lidar in Oberpfaffenhofen on October 17 of 2017. Geometric average of all SNR estimates (black solid curve) and maximal and minimal values of the signal-to-noise ratio (dashed curves); relative errors $E_{V}$ and probabilities $P_{<2}$ in the cases of application of the FSWF and MFAS methods (blue and red curves, respectively).

Figure 5 shows the distributions of the wind speed and direction upon application of the procedure described by Eqs. (15) and (16). This procedure allows significant improvement of the result (cp. Fig.4(c,d) and Fig.5). It can be seen from Fig. 5 that sharp changes of estimate of the wind speed and direction mostly take place in a rectangle with the sides 1500-2000 $\mathrm{m}$ and 6-18 h. It follows from Fig.5 that the obtained distributions for the wind are rather smooth for heights no larger than $1445 \mathrm{~m}$, which were used as reference (true) ones. Just as in the numerical experiment, we used the results of lidar measurements during one day (October 17 of 2017) to calculate the probability $P_{<2}$ that the conditions 
$\left|\hat{V}_{x}^{(\mathrm{n})}-\bar{V}_{x}\right|<2 \mathrm{~m} / \mathrm{s}$ and $\left|\hat{V}_{y}^{(\mathrm{n})}-\bar{V}_{y}\right|<2 \mathrm{~m} / \mathrm{s}$ are fulfilled simultaneously, as well as the standard error of estimation of the wind velocity vector determined as $E_{V}=\sqrt{\left\langle\left(\hat{V}_{x}^{(\mathrm{n})}-\bar{V}_{x}\right)^{2}+\left(\hat{V}_{y}^{(\mathrm{n})}-\bar{V}_{y}\right)^{2}\right\rangle}$, where $\left\{\hat{V}_{x}, \hat{V}_{y}\right\}$ is the estimate obtained for one scan. The index $n=1,2,3$ corresponds to the DSWF, FSWF, and MFAS methods, respectively. Since the DSWF method is less efficient than FSWF and MFAS, we compare the accuracies of the two last methods.

Figure 6 shows the vertical profiles of the signal-to-noise ratio SNR, the error $E_{V}$, and the probability $P_{<2}$ in the cases of application of FSWF (blue curves) and MFAS (red curves). One can see that reliable (with the probability $P_{<2}$ not smaller than 99\%) estimates of the wind velocity vector take place at SNR $>-27 \mathrm{~dB}$ (according to the black solid curve) in the case of FSWF and at SNR $>-30 \mathrm{~dB}$ in the case of MFAS. This result is in a good agreement with the results of numerical experiment (see Table 2). Since DSWF can provide an acceptable result only at SNR $\geq-20 \mathrm{~dB}$, the application of DSWF for processing of lidar data in that day allowed retrieval of the vertical profiles of wind, on average, up to a height of $700 \mathrm{~m}$ with allowance for the signal-to-noise ratio shown by the black curve in Fig.6. At the same time, in the case of application of the FSWF and MFAS methods, the wind profiles can be retrieved up to heights of $1000 \mathrm{~m}$ and $1300 \mathrm{~m}$, respectively. This analysis has shown that the MFAS method is most efficient among the considered methods: the excess of the maximum height of retrieval of wind vector profiles by this method is, on average, $600 \mathrm{~m}$ (86\%) in comparison with DSWF and $300 \mathrm{~m}(30 \%)$ in comparison with FSWF.

\section{SUMMARY}

Thus, in this paper, we have studied the efficiency of the MFAS method for estimation of the wind velocity vector from measurements by Windcube 200s MPCDL under conditions of a weak echo signal in comparison with the DSWF and FSWF methods. In the numerical experiment, it was found that the MFAS method allows an acceptable result to be obtained at the signal-to-noise ratio below the limits of applicability of FSWF by $2.5 \mathrm{~dB}$ and DSWF by $10 \mathrm{~dB}$. The analysis of results of the field experiment has shown that the maximum height of reconstruction of vertical profiles of the wind speed and direction by the DSWF, FSWF, and MFAS methods is, on average, $700 \mathrm{~m}, 1000 \mathrm{~m}$, and $1300 \mathrm{~m}$.

A disadvantage of the MFAS method is that it requires much longer computer time in comparison with DSWF and FSWF. However, the combination of these three methods (for example, for the conditions of the field experiment considered above, it is possible using DSWF at $\mathrm{S} \bar{N} R \geq-18 \mathrm{~dB}$, FSWF at $-18 \mathrm{~dB}>\mathrm{S} \bar{N} R \geq-24 \mathrm{~dB}$, and MFAS at $-33 \mathrm{~dB} \leq \mathrm{S} \overline{\mathrm{N}} \mathrm{R}<-24 \mathrm{~dB}$ ) allows us to shorten the time for retrieval of vertical profiles of the wind speed and direction by almost an order of magnitude and to obtain the information about the wind in real time.

Acknowledgments: This study was supported by the Russian Foundation for Basic Research, Project No. 16-42-700072.

\section{REFERENCES}

[1] Lhermitte, R. M. and Atlas, D., "Precipitation motion by pulse Doppler," Proceedings of the $9^{\text {th }}$ Weather Radar Conference. Kansas City, MO, USA. 218-223 (1961).

[2] Doviak, R. J. and Zrnic, D. S., [Doppler radar and weather observations], Academic Press. San Diego. 1- 458 (1984).

[3] Smalikho, I., "Techniques of wind vector estimation from data measured with a scanning coherent Doppler lidar," Journal of Atmospheric and Oceanic Technology 20(2), 276-291 (2003).

[4] Vasiljevic, N., Lea, G., Courtney, M., Cariou, J.-P., Mann, J., and Mikkelsen, T., "Long-Range WindScanner System," Remote Sensing 8(11), 896 (2016); doi:10.3390/rs8110896

[5] Banakh, V. A. and Smalikho, I. N., [Coherent Doppler wind lidars in a turbulent atmosphere], Artech House, Boston \& London, 1-248 (2013).

[6] Salamitou, P., Dabas, A., and Flamant, P. H., "Simulation in the time domain for heterodyne coherent laser radar," Applied Optics 34(3), 499-506 (1995).

[7] Frehlich, R. G., "Effect of wind turbulence on coherent Doppler lidar performance," Journal of Atmospheric and Oceanic Technology 14(2), 54-75 (1997). 